\title{
NECESSARY AND SUFFICIENT CONDITIONS FOR FACTORABILITY OF NONNEGATIVE OPERATOR- VALUED FUNCTIONS ON A BANACH SPACE
}

\author{
A. G. MIAMEE AND H. SALEHI ${ }^{1}$
}

ABSTRACT. Several necessary and sufficient conditions for factorability of positive operator-valued functions on a Banach space are given. These characterizations involve the analysis of quasi square root and their corresponding invariant subspaces. The work extends to the Banach space a recent theorem of Yu. A. Rozanov and a certain earlier result of $R$. Payen. It also reveals the connection between their works on the factorization problem.

1. Introduction. The problem of factoring nonnegative operator-valued functions on a Banach space plays an important role in the study of Banach space valued stationary stochastic processes [1]. In [4] we were able to extend to the Banach space case the work of R. G. Douglas [2] on factoring nonnegative operator-valued functions. However these results provide only sufficient conditions for the factorization problem. Our purpose here is to establish some necessary and sufficient conditions for factorability of nonnegative operator-valued functions on a Banach space. This extends to the Banach space the recent work of Yu. A. Rozanov [6] and a certain earlier result of $R$. Payen [5] on necessary and sufficient conditions for the factorization problem. It also reveals the close connection which exists between these characterizations.

When $f$ is a positive operator-valued function on a Hilbert space, $\sqrt{ } f$, the square root of $f$ whose existence is known, plays a significant role. But when $f$ is a positive operator-valued function on a Banach space $X$, the existence of a square root in the ordinary way does not make sense. Nevertheless, we have recently proved [4] the existence of a measurable operatorvalued function $Q$ on $X$ into some auxiliary Hilbert space which behaves

Received by the editors August 14, 1973.

AMS (MOS) subject classifications (1970). Primary 42A88, 42A96; Secondary 60G10.

Key words and phrases. Banach space, positive operator-valued functions, quasi square root, factorability, regularity, stationary processes.

${ }^{1}$ Supported in part by NSF GP-28658. 
almost like a square root in the sense that $f=Q^{*} Q$. The operator-valued function $Q$ is called a quasi square root.

In $\$ 2$ we set up necessary terminology and state some known results. In $\$ 3$ we prove our main theorem on characterizing factorable positive operator-valued functions on a Banach space. In establishing our result we make use of quasi square root and techniques employed in [6].

2. Preliminaries. Let $\mathcal{K}$ be a separable Hilbert space and let $L_{2}(\mathcal{K})$ denote the Hilbert space of all $K$-valued functions defined on the unit circle that are weakly measurable and have square summable norms with respect to (w.r.t.) Lebesgue measure. The $L_{2}(\mathcal{K})$ inner product of two functions $g_{1}$ and $g_{2}$ is given by

$$
\frac{1}{2 \pi} \int_{0}^{2 \pi}\left(g_{1}\left(e^{i \theta}\right), g_{2}\left(e^{i \theta}\right)\right) d \theta
$$

The operator $U$, defined to be multiplication by $e^{i \theta}$ on $L_{2}(\mathcal{K})$, is unitary and a shift. The subspace $L_{2}^{0+}(\mathcal{K})\left(L_{2}^{0-}(\mathcal{K})\right.$ consisting of those functions $g$ in $L_{2}(\mathcal{K})$ for which $\int_{0}^{2 \pi} g\left(e^{i \theta}\right) e^{i n \theta} d \theta=0$ for $n>0(n<0)$ is an invariant subspace of $U\left(U^{*}\right)$ 。

Let $X$ be a Banach space. $X^{*}$ denotes the space of all bounded conjugate linear functionals on $\mathcal{X}$. For any two Banach spaces $X$ and $\mathcal{Y}$, $B(\mathcal{X}, \mathcal{Y})$ denotes the class of all bounded linear operators on $\mathcal{X}$ into $\mathcal{Y}$. $\mathrm{A}$ weakly measurable $B(X, K)$-valued function $A=A\left(e^{i \theta}\right)$ is said to be analytic if for each $x \in X, A\left(e^{i \theta}\right) x \in L_{2}^{0-}(\mathcal{K})$. From now on it is assumed that the Banach space $X$ is separable.

2.1. Definition. Let $f$ be in $B\left(X, X^{*}\right) . f$ is said to be nonnegative if for each $x$ in $X,(f x)(x) \geq 0 . B^{+}\left(X, X^{*}\right)$ denotes the class of all nonnegative operators on $X$ into $X^{*}$.

2.2. Definition. Let $f=f\left(e^{i \theta}\right)$ be a weakly summable $B^{+}\left(\mathcal{X}, X^{*}\right)$-valued function on the unit circle. We say that $f$ is factorable if there exists a Hilbert space $\mathcal{K}$ and an analytic $B(\mathcal{X}, \mathcal{K})$-valued function $A=A\left(e^{i \theta}\right)$ such that $f\left(e^{i \theta}\right)=A^{*}\left(e^{i \theta}\right) A\left(e^{i \theta}\right)$, in the sense that $\left(f\left(e^{i \theta}\right) x\right)(y)=\left(A\left(e^{i \theta}\right) x\right.$, $\left.A\left(e^{i \theta}\right) y\right) ; x, y \in X$.

We now state the following crucial lemma which is proved in [4].

2.3. Lemma. Let $f$ be a weakly summable $B^{+}\left(X, X^{*}\right)$-valued function on the unit circle. Then there exists a separable Hilbert space $\mathcal{K}$ and $a$ measurable $B(\mathcal{X}, \mathcal{K})$-valued function $Q=Q\left(e^{i \theta}\right)$ on the unit circle such that

$$
f\left(e^{i \theta}\right)=Q^{*}\left(e^{i \theta}\right) Q\left(e^{i \theta}\right)
$$


in the sense that

$$
\left(f\left(e^{i \theta}\right) x\right)(y)=\left(Q\left(e^{i \theta}\right) x, Q\left(e^{i \theta}\right) y\right) ; \quad x, y \in X .
$$

The above lemma allows us to make the following

2.4. Definition. The function $Q=Q\left(e^{i \theta}\right)$ in Lemma 2.3 is called a quasi square root of $f$.

In the absence of the square root, a quasi square root has almost all the desired properties. One of its applications is demonstrated in Theorem 3.8 where necessary and sufficient conditions for factorability of $f$ are given.

We now make a digression to state some necessary terminologies concerning Banach space valued stationary stochastic processes. Let $X$ be a separable Banach space and $\mathcal{K}$ be a separable Hilbert space. By a stationary stochastic process (SSP) we mean a $B(\mathcal{X}, \mathcal{K})$-valued sequence $\xi_{n}$, $-\infty<n<\infty$, of bounded linear operators on $\mathcal{X}$ into $\mathcal{K}$ such that $\xi_{n}^{*} \xi_{m}$ depends only on $m-n$. The operator $R(m-n)=\xi_{n}^{*} \xi_{m}$ is called the covariance operator of the process. It is known [1] that we have the representation

$$
R(m-n)=\frac{1}{2 \pi} \int_{0}^{2 \pi} e^{-i(m-n) \theta} d F\left(e^{i \theta}\right),
$$

where the spectral distribution $F$ is a measure on the Borel sets of the unit circle with values in $B^{+}\left(X, X^{*}\right)$, and the above integral is taken in the weak sense. When $F$ is absolutely continuous (a.c.) w.r.t. Lebesgue measure, its density $f$ is a bilinear functional on $X \times X . f$ is called the spectral density of the process. We denote by $H_{\xi}(n)$ the subspace of $\mathcal{K}$ spanned by all $\xi_{k} x,-\infty<k \leq n, x \in \mathcal{X}$ and set $H_{\xi}(-\infty)=\bigcap_{n} H_{\xi}(n)$. We say that the process is regular if $H_{\xi}(-\infty)=\{0\}$.

Let $f=f\left(e^{i \theta}\right)$ be a weakly summable $B^{+}\left(X, X^{*}\right)$-valued function on the unit circle. Then by Lemma 2.3 a quasi square root of $f, Q=Q\left(e^{i \theta}\right)$, with values in $B(\mathcal{X}, \mathcal{K})$ exists. Let $\xi_{n}=e^{i n} \theta(\theta)$. Then $\xi_{n},-\infty<n<\infty$, is a $B\left(X, L_{2}(\mathcal{K})\right.$ )-valued SSP whose spectral density is $f$. From here on $\xi_{n}$, $-\infty<n<\infty$, represents this process.

In $\$ 3$ we need Lemma 2.5 contained in [6], which can also be essentially obtained from Theorem 8 of [3]. First we introduce some notations (c.f. [6]). Let $B$ be a linear manifold in $L_{2}(\mathcal{K})$ and $S=\left\{g_{n}\left(e^{i \theta}\right)\right\}_{n=1}^{\infty}$ be a complete orthonormal system of functions in $B$. We denote by $B_{\mathcal{S}}\left(e^{i \theta}\right)$ the linear manifold in the Hilbert space $\mathcal{K}$ generated by all values $g_{1}\left(e^{i \theta}\right)$, $g_{2}\left(e^{i \theta}\right), \cdots$. Obviously the closure $\overline{\bar{B}}\left(e^{i \theta}\right)=\overline{\bar{B}}_{\mathcal{S}}\left(e^{i \theta}\right)$ does not depend on $\mathcal{S}$ in the sense that $\overline{\bar{B}}_{\mathcal{S}_{1}}\left(e^{i \theta}\right)=\overline{\bar{B}}_{\mathcal{S}_{2}}\left(e^{i \theta}\right)$ a.e. $\theta$, if $\mathcal{S}_{1}$ and $\mathcal{S}_{2}$ are any two complete orthonormal systems in $B$. 
In case $B=Q\left(e^{i \theta}\right) \mathcal{X}$ it easily follows that a.e.

$$
\overline{\bar{B}}\left(e^{i \theta}\right)=\overline{Q\left(e^{i \theta}\right) X} \text { a.e., }
$$

where $\overline{Q\left(e^{i \theta}\right) \mathcal{X}}$ denotes the closure of the range of the operator $Q\left(e^{i \theta}\right)$.

2.5. Lemma. Let $B$ be a linear manifold in $L_{2}(\mathcal{K})$. Then the subspace $\widetilde{G}\left(e^{\text {in } \theta_{B}},-\infty<n<\infty\right)$ generated by $e^{i n \theta} B,-\infty<n<\infty$, consists of all functions $g \in L_{2}(\mathcal{K})$ such that $g\left(e^{i \theta}\right) \in \overline{\bar{B}}\left(e^{i \theta}\right)$ a.e.

3. Main results. In this section we prove our main results. $X$ is a separable Banach space and $\mathcal{K}$ is a separable Hilbert space. $f$ is a weakly summable $B^{+}\left(X, X^{*}\right)$-valued function on the unit circle. $Q$ will denote a quasi square root of $f$ with values in $B(\mathcal{X}, \mathcal{K}) . \xi_{n}=e^{i n} \theta Q\left(e^{i \theta}\right),-\infty<n<\infty$, is a $B\left(\mathcal{X}, L_{2}(\mathcal{K})\right)$-valued SSP with the spectral density $f$. Let $H=\widetilde{G}\left(\xi_{n} x\right.$,

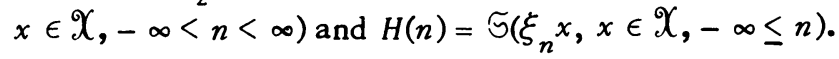

We shall be interested in the structure of the subspaces $B=H(T)$ $H(S)$, where $T, S$ are some sets of integers and for any set $T, H(T)=$ $\widetilde{S}\left(\xi_{n} x, x \in X, n \in T\right)$. One can say that $B$ is the innovation of $H(T)$ in comparison with $H(S)$.

$L_{T}$ will denote the linear space of all $X^{*}$-valued integrable functions $\phi$ \{for each $x \in X, \phi\left(e^{i \theta}\right) x$ is summable $\}$ with Fourier decomposition of the form

$$
\phi\left(e^{i \theta}\right) \sim \sum_{n \in T} a_{n} e^{n i \theta}, \quad a_{n} \in X^{*}
$$

i.e.

$$
\phi\left(e^{i \theta}\right) x \sim \sum_{n \in T} a_{n} x e^{n i \theta}, \quad x \in X
$$

such that

$$
\phi\left(e^{i \theta}\right) \in Q^{*}\left(e^{i \theta}\right) \mathcal{K}
$$

and

$$
\int_{0}^{2 \pi}\left\|Q^{*-1}\left(e^{i \theta}\right) \phi\left(e^{i \theta}\right)\right\|^{2} d \theta<\infty
$$

where $Q^{*-1}\left(e^{i \theta}\right)$ is the inverse operator from $Q^{*}\left(e^{i \theta}\right) \mathcal{K}$ onto $\Re_{Q^{*}\left(e^{i \theta}\right)}^{\perp}=$ orthogonal complement of the null subspace of $Q^{*}\left(e^{i \theta}\right)$.

3.4. Lemma. Let $S$ be the complement of $T$ in the set of all integers and $B_{T}=H(T) \ominus H(S)$. Then

$$
B_{T}=Q^{*-1} L_{T}
$$


Proof. Let $\phi\left(e^{i \theta}\right) \in B_{T^{*}}$. Define $\Psi\left(e^{i \theta}\right)=Q^{*}\left(e^{i \theta}\right) \phi\left(e^{i \theta}\right)$. From the relations

$$
\begin{aligned}
\int_{0}^{2 \pi}\left(Q^{*}\left(e^{i \theta}\right) \phi\left(e^{i \theta}\right)\right)(x) d \theta & =\int_{0}^{2 \pi}\left(\phi\left(e^{i \theta}\right), Q\left(e^{i \theta}\right) x\right) d \theta \\
& \leq\left(\int_{0}^{2 \pi}\left\|\phi\left(e^{i \theta}\right)\right\|^{2} d \theta\right)^{1 / 2}\left(\int_{0}^{2 \pi}\left\|Q\left(e^{i \theta}\right) x\right\|^{2} d \theta\right)^{1 / 2}<\infty,
\end{aligned}
$$

it follows that $\Psi\left(e^{i \theta}\right)(x), x \in X$, is summable. Also we have that

$$
\begin{aligned}
0 & =\int_{0}^{2 \pi} e^{-i s \theta}\left(\phi\left(e^{i \theta}\right), Q\left(e^{i \theta}\right) x\right) d \theta \\
& =\int_{0}^{2 \pi} e^{-i s \theta}\left(Q^{*}\left(e^{i \theta}\right), \phi\left(e^{i \theta}\right)\right)(x) d \theta, \quad s \in \mathcal{S} .
\end{aligned}
$$

Since $\phi \in H$, by Lemma 2.5 and the paragraph preceding it, $\phi\left(e^{i \theta}\right) \epsilon$ $Q\left(e^{i \theta}\right) X$ a.e. Hence

$$
\phi\left(e^{i \theta}\right)=Q^{*-1}\left(e^{i \theta}\right) \Psi\left(e^{i \theta}\right) \text { and } \Psi\left(e^{i \theta}\right) \in L_{T^{*}}
$$

Thus $B_{T} \subseteq Q^{*-1} L_{T^{*}}$ Now let $\Psi\left(e^{i \theta}\right)$ be in $L_{T^{*}}$ Set $\phi\left(e^{i \theta}\right)=Q^{*-1}\left(e^{i \theta}\right) \Psi\left(e^{i \theta}\right)$. We will show that $\phi\left(e^{i \theta}\right) \in B_{T}$. We note that for each $x \in \mathcal{X},\left(\phi\left(e^{i \theta}\right)\right.$, $\left.Q\left(e^{i \theta}\right) x\right)=\Psi\left(e^{i \theta}\right)(x)$. Hence

$$
\int_{0}^{2 \pi} e^{-i s \theta}\left(\phi\left(e^{i \theta}\right), Q\left(e^{i \theta}\right) x\right) d \theta=0, \quad s \in \mathcal{S}, x \in X .
$$

It suffices to show that $\phi \in H=\widetilde{S}\left(e^{i n \theta} Q\left(e^{i \theta}\right) X,-\infty<n<\infty\right)$. But $\phi\left(e^{i \theta}\right) \epsilon$ range of $Q^{*-1}\left(e^{i \theta}\right)=\overline{Q\left(e^{i \theta}\right) X}$. Hence by Lemma 2.5 and the paragraph preceding it, we have that $\phi\left(e^{i \theta}\right) \in B_{T}$. This completes the proof of $Q^{*-1} L_{T}$ $\subseteq B_{T}$ only if

3.6. Corollary. The relation $\mathcal{S}\left(e^{i n} B_{T},-\infty<n<\infty\right)=H$ bolds if and

Proof. Since $H=\widetilde{G}\left(e^{i n \theta} Q\left(e^{i \theta}\right) X,-\infty<n<\infty\right)$, by Lemma 2.5, $H=$ $\widetilde{G}\left(e^{i n \theta} B_{T},-\infty<n<\infty\right)$ if and only if $\overline{\bar{B}}_{T}\left(e^{i \theta}\right)=\overline{Q\left(e^{i \theta}\right) X}$. But by the last lemma $\overline{\bar{B}}_{T}\left(e^{i \theta}\right)=\overline{\overline{Q^{*-1} L_{T}}}\left(e^{i \theta}\right)$.

We note that this result in particular is useful for regularity if we take $T$ to be the set of all nonnegative integers. In this case the space $L_{T}$ is included in the Hardy class of functions $\phi\left(e^{i \theta}\right) \sim \Sigma_{0}^{\infty} a_{n} e^{i n \theta}$. More precisely they are so that for each $x \in X$, the scalar-valued function $\phi\left(e^{i \theta}\right)(x)$ is in the classical Hardy class $H^{1}$. We also note that in this case the rela- 
tion $\widetilde{S}\left(e^{i n \theta_{B}}{ }_{T},-\infty<n<\infty\right)=H$ of Corollary 3.6 is equivalent to the regularity of the process $\xi_{n}=e^{i n \theta} Q\left(e^{i \theta}\right),-\infty<n<\infty$.

We are now ready to prove our main result.

3.8. Theorem. Let $f=f\left(e^{i \theta}\right)$ be a weakly summable $B^{+}\left(X, X^{*}\right)$-valued function on the unit circle. Then the following statements are equivalent:

1. $f$ is factorable.

2. There exists an analytic $B(\mathcal{X}, \mathcal{K})$-valued function $\Psi$ such that

(i) $\Psi^{*}\left(e^{i \theta}\right) \mathcal{K} \subseteq Q^{*}\left(e^{i \theta}\right) \mathcal{K}$;

(ii) $\overline{Q^{*-1}\left(e^{i \theta}\right) \Psi^{*}\left(e^{i \theta}\right) \mathcal{K}}=\overline{Q\left(e^{i \theta}\right) X}$ a.e.;

(iii) $\int_{0}^{2 \pi}\left\|Q^{*-1}\left(e^{i \theta}\right) \Psi^{*}\left(e^{i \theta}\right) k\right\|^{2} d \theta<\infty, k \in \mathcal{K}$.

3. The process $e^{i n} \theta\left(e^{i \theta}\right),-\infty<n<\infty$, is regular.

4. There exists a sequence $\left\{\phi_{n}\left(e^{i \theta}\right)\right\}_{n=1}^{\infty}$ of measurable K-valued functions such that

(a) a.e. $\theta,\left\{\phi_{n}\left(e^{i \theta}\right)\right\}_{n=1}^{\infty}$ is an orthonormal bas is for $\overline{Q\left(e^{i \theta}\right) X}$;

(b) for each $n, g_{n}\left(e^{i \theta}\right) x=Q^{*}\left(e^{i \theta}\right) \phi_{n}\left(e^{i \theta}\right) x$ is in the usual Hardy class $H^{2}$ for all $x \in X$.

Proof. (1) $\Rightarrow(2)$. Let $f=\Phi^{*} \Phi$, where $\Phi$ is analytic. Then

$$
\left\|Q\left(e^{i \theta}\right) x\right\|^{2}=\left\|\Phi\left(e^{i \theta}\right) x\right\|^{2}, \quad x \in X
$$

Define

$$
V\left(e^{i \theta}\right) Q\left(e^{i \theta}\right) x=\Phi\left(e^{i \theta}\right) x, \quad x \in X
$$

By (3.9)-(3.10), $V\left(e^{i \theta}\right)$ can be extended to an isometry on $\overline{Q\left(e^{i \theta}\right) X}$ onto $\overline{\Phi\left(e^{i \theta}\right) X}$. Then the operator-valued function $\Psi=V Q$ satisfies the conditions (i)-(iii) of (2).

$(2) \Rightarrow(3)$. Let $\Psi$ be an analytic $B(\mathcal{X}, \mathcal{K})$-valued function satisfying (i)-(iii). By Corollary 3.6 and the paragraph following this corollary, it suffices to show that $\overline{Q\left(e^{i \theta}\right) \bar{X}}=\overline{\overline{Q^{*-1} L_{T}}}\left(e^{i \theta}\right)$ a.e. Clearly the right-hand side is a subset of the left-hand side. The other inclusion follows from (ii)-(iii) and the fact that for each $k \in \mathcal{K}, \Psi^{*} k \in L_{T^{*}}$

(3) $\Rightarrow(4)$. Since $e^{i n \theta} Q\left(e^{i \theta}\right),-\infty<n<\infty$, is a regular process, $H(0)=$ $\widetilde{S}\left(e^{i n \theta} Q\left(e^{i \theta}\right) \mathcal{X}, n \leq 0\right)$ does not contain a nontrivial doubly invariant subspace of $\mathcal{K}$. Hence by $\left[3\right.$, p. 61] it is of the form $V L_{2}^{0-}(\mathcal{H})$, where $V$ is a measurable isometry operator on some Hilbert space $\mathcal{H}$ into $\mathcal{K}$. Let $\left\{\phi_{n}\left(e^{i \theta}\right)\right\}_{n=1}^{\infty}$ be an orthonormal basis for $H(0) \ominus H(-1)$. An argument similar to one used in $[5$, p. 380] and $[3$, p. 61] may be used to show that for 


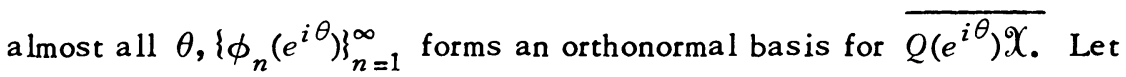
$x \in X$. To show $g_{n}\left(e^{i \theta}\right)(x)=Q^{*}\left(e^{i \theta}\right) \phi_{n}\left(e^{i \theta}\right)(x)$ is in the Hardy class $H^{2}$, we observe that for each $n, \phi_{n} \perp e^{i k \theta} Q\left(e^{i \theta}\right) x$ for $x \in X$ and $k \leq-1$. Hence

$$
\begin{aligned}
\int_{0}^{2 \pi} e^{-i k \theta} g_{n}\left(e^{i \theta}\right)(x) d \theta & =\int_{0}^{2 \pi} e^{-i k \theta} Q^{*}\left(e^{i \theta}\right) \phi_{n}\left(e^{i \theta}\right)(x) d \theta \\
& =\int_{0}^{2 \pi} e^{-i k \theta}\left(\phi_{n}\left(e^{i \theta}\right), Q\left(e^{i \theta}\right) x\right) d \theta=0 .
\end{aligned}
$$

Thus $g_{n} x \in H^{2}$.

$(4) \Rightarrow(1)$. Let (a)-(b) hold. Let $\left\{e_{n}\right\}_{n=1}^{\infty}$ be an orthonormal basis for K. We define the operator-valued function $\Phi$ by

$$
\Phi\left(e^{i \theta}\right) x=\sum_{n} \overline{g_{n}\left(e^{i \theta}\right)}(x) e_{n}, \quad x \in X .
$$

We note that for all $x, y \in X$,

$$
\begin{aligned}
(f x)(y) & =(Q x, Q y)=\sum_{n}\left(Q x, \phi_{n}\right) \overline{\left(Q y, \phi_{n}\right)} \\
& \left.=\sum_{n} \overline{\left(Q^{*} \phi_{n} x\right.}\right)\left(Q^{*} \phi_{n} y\right)=\sum_{n} \overline{\left(g_{n} x\right)}\left(g_{n} y\right) \\
& =\left(\sum_{n} \overline{\left(g_{n} x\right)} e_{n}, \sum_{n}\left(\overline{\left(g_{n} y\right)} e_{n}\right)=(\Phi x, \Phi y),\right.
\end{aligned}
$$

where the second equality follows by (a). Hence $\Phi\left(e^{i \theta}\right) \in B(\mathcal{X}, \mathcal{K})$. Because of weak summability of $f$ it follows that for each $x \in \mathcal{X}, \Phi x \in L_{2}(\mathcal{K})$. Using (3.11) and (3.12) it is not hard to show that the analytic $B(\mathcal{X}, \mathcal{K})$ valued sequence $\Phi_{N}\left(e^{i \theta}\right) x=\sum_{n=1}^{N} g_{n}\left(e^{i \theta}\right)(x) e_{n}$ converges to $\Phi\left(e^{i \theta}\right) x$ in $L_{2}(\mathcal{K})$. Therefore $\Phi \in L_{2}^{0}=(\mathcal{K})$. Since by (3.12), $f=\Phi^{*} \Phi$, the result follows.

We remark that if one assumes $\int_{0}^{2 \pi}\left\|f\left(e^{i \theta}\right)\right\| d \theta<\infty$, then part (b) of (4) in the above theorem can be replaced by $\left(b^{\prime}\right)$ : For each $n, g_{n}\left(e^{i \theta}\right)=$ $Q^{*}\left(e^{i \theta}\right) \phi_{n}\left(e^{i \theta}\right)$ satisfies $\int_{0}^{2 \pi}\left\|g_{n}\left(e^{i \theta}\right)\right\|^{2} d \theta<\infty$.

\section{REFERENCES}

1. S. A. Cobanjan, Regularity of stationary processes with values in a Banach space and factorization of operator-valued functions, Sakharth. SSR Mech. Akad. Moambe 61 (1971), 29-32. (Russian) MR 44 \#7631.

2. R. G. Douglas, On factoring positive operator functions, J. Math. Mech. 16 (1966), 119-126. MR 35 \#782. 
3. H. Helson, Lectures on invariant subspaces, Academic Press, New York, 1964. MR $30 \# 1409$.

4. A. G. Miamee and H. Salehi, Factorization of positive operator valued functions on a Banach space, Indiana Univ. Math. J. (to appear).

5. R. Payen, Fonctions aléatoires du second ordre à valeurs dans un espace de Hilbert, Ann. Inst. H. Poincaré Sect. B 3 (1967), 323-396. MR 37 \#6993.

6. Ju. A. Rozanov, Some approximation problems in the theory of stationary processes, J. Multivariate Anal. 2 (1972), 135-144. MR 46 \#4597.

DEPARTMENT OF MATHEMATICS, MICHIGAN STATE UNIVERSITY, EAST LANSING, MICHIGAN 48823 (Current address of H. Salehi)

Current address (A. G. Miamee): Department of Mathematics, Arya-Mehr University of Technology, Tehran, Iran 